this study was to use functional magnetic resonance imaging (fMRI) to identify the neural circuits underlying disturbances processing oddball stimuli in firstepisode schizophrenia (FES).

Method: fMRI data were collected from 24 people with FES (within 3 months of service contact) and 24 matched healthy controls while performing an auditory oddball task comprising $15 \%$ target (high) tones and $85 \%$ standard (low) tones. Data were analyzed in SPM2, with event-related analysis of the supramarginal gyrus, thalamus, and limbic and prefrontal cortical areas.

Results: The FES group showed significantly reduced activity in the thalamus, hippocampus, dorsal lateral prefrontal cortex and supramarginal gyrus, but a pattern of enhancement as well as reduction in medial prefrontal/anterior cingulate activity, compared with controls.

Conclusions: These findings suggest that schizophrenia is associated with impairments in networks for processing salience as well as context from the first episode of this illness. Dysregulation of medial prefrontal areas may reflect an attempt to compensate for a fundamental breakdown in the coordination of these processes.

\section{Functional brain imaging of auditory prepulse inhibition}

\section{Campbell, TW Budd, R Fulham, M Hughes, F Karayanidis, M-C Hanlon, W Stojanov, P Johnston, U Schall}

University of Newcastle, Newcastle, Australia

Aims/Background: Inhibition deficits are consistently shown in a broad spectrum of neuropsychiatric conditions, such as schizophrenia, implicating altered dopamine neurotransmission. This results in impaired 'sensorimotor gating', a physiological measure of inhibitory brain processes. We investigated the neural networks underlying sensorimotor gating.

Methods: Sensorimotor gating was measured using prepulse inhibition (PPI) of the acoustic startle eyeblink response through bipolar EMG from the left orbicularis oculi muscle and functional magnetic resonance imaging (fMRI). fMRI images were acquired using sparse temporal sampling techniques to minimize stray masking noise interfering with the auditory processing. Trial types: (A) startle probes alone (baseline), $(\mathrm{B} / \mathrm{C})$ startle probes preceded by a prepulse at 120 or $480 \mathrm{~ms}$ and (D) 'scanner-noise only'. Significant BOLD contrasts were assessed using one-sample $t$-tests $[P>0.001$ (uncorrected) and exploratory data analyses at $P>0.01]$.
Results: Data from 16 healthy volunteers (9 men:7 women, age: $23 \pm 4$ years) were included. Cortical peak BOLD activation was confirmed for the superior temporal (STG), inferior frontal (IFG), precentral gyri and quadrangularis lobule for the baseline vs. scannernoise only. No changes were detected for the STG and IFG in baseline vs. 120 or $480 \mathrm{~ms}$ prepulse condition, whereas increased right IFG activation was confirmed for the short vs. long lead interval contrast. Explorative analyses suggested concurrent decrease of activation in the right anterior STG in the 120 vs. 480 ms condition. When correlating the electromyographically recorded PPI effect with hemodynamic responses, the activation was mediated by thalamic activation (bilateral).

Conclusions: Thalamic activation mediates auditory PPI. The potential relationship with impaired sensorimotor gating in clinical populations remains to be investigated.

\section{Values of cross-cultural research: insights gained from the validation of the 'Depression Screening Measure DMI-10 Chinese version'}

\section{B Chan ${ }^{1,2}$, G Parker ${ }^{1,2}$ \\ 'School of Psychiatry, University of New South Wales; and ${ }^{2}$ The Black Dog Institute, Sydney, Australia}

Background: Depression measurement tools in crosscultural research require careful design and thorough validation to ensure that cognitive concepts in one culture can be appropriately translated and applied to a differing culture. The aim of this paper was to report the validation of the Chinese version of a screening measure of state depression, the DMI- 10.

Method: Three interdependent studies were conducted: 1) an initial bilingual test-retest study identified four (of the ten) items as having poor cross-cultural validity, 2) a second study involved focus group participants exploring the meaning of translated items with Chinese speakers and 3) the third study repeated the bilingual test-retest analyses on the modified DMI- 10 .

Results: Study 3 showed improved correlation coefficients on all items and an excellent overall correlation $(r=0.87)$ between the Chinese and English versions.

Conclusions: The findings have significant implications for cross-cultural research. Subsequently, our research team undertook a study to validate the Chinese DMI-10 against the Chinese versions of the BDI-II and the CIDI. The DMI-10 should prove useful in identifying Chinese people at risk of clinical depression. 